\title{
Collabohab: A Technology Probe into Peer Involvement in Cardiac Rehabilitation
}

\author{
Julie Maitland \\ University of Glasgow, 17 Lilybank Gardens, Glasgow G12 8QQ, UK \\ jules@dcs.gla.ac.uk
}

\begin{abstract}
Mobile and ubiquitous systems designed to promote an increase in physical activity by harnessing social influence have so far had variable success. Taking a cardiac rehabilitation program as a specific health domain, in which physical inactivity is one of several targeted behaviours, the research described in this paper aims to elicit understanding of peer-involvement in health-related behavioural change and explore the potential for effective technological support. This paper introduces the technology probe Collabohab and discusses the accompanying methodological approach being adopted to establish insight into the important but so far little understood phenomenon of social support within health-related behavioural change.
\end{abstract}

Keywords: health, cardiac rehabilitation, social support, technology probe.

\section{Introduction}

Physical inactivity is a widespread problem. The World Health Organisation estimates that $60 \%$ of the worldwide population does not achieve the minimum recommended level of activity [1]. Over recent years the problem of physical inactivity has received much interest from computer scientists in both the academic and commercial domain. The ever-increasing ability of pervasive technology to detect the nature of an individual's movements lends itself well to the established behavioural change technique (BCT) of self-monitoring [2], and has led to the development of many physical activity promoting applications [3-5]. The evolution of single-user activity promotion systems into multi-user equivalents seems to be a natural progression in this area of research, as the inclusion of social support promises to increase the likelihood of increased and prolonged physical activity. However, previous studies of multi-user activity promotion systems, including our own, have generated diverse and sometimes conflicting results [3-6]. In response to these findings we have decided to explore the potential of social support within activity promotion systems further. Unlike the previous work already mentioned, in which the systems were designed for and evaluated with relatively fit and healthy individuals, we focus on individuals who have a vested interest in increasing their daily activity levels: individuals enrolled on a cardiac rehabilitation program.

The planned study aims to investigate peer involvement in an existing cardiac rehabilitation program while exploring the potential for supportive technological 
interventions - systems that support appropriate peer-involvement in rehabilitation programs. By designing and deploying a technology probe into a rehabilitation program we aim to determine the information needs of rehabilitation participants and their peers, and to discover what level of involvement they feel is appropriate. By discovering how, when, why and with whom rehab participants wish to share information, steps can be taken towards developing technological means of supporting the different levels and dimensions of peer involvement and social support.

This paper will begin by providing a brief overview of the role of social support within health and behavioural change applications. We will then describe the methodological approach being taken in this work and introduce the technology probe, Collabohab, which has been designed for the purpose of the planned study.

\section{Background}

Social support is widely acknowledged as having a positive relationship with many health related outcomes [7-9], and within rehabilitation social support is considered a resource for behavioural change [2]. Despite such wide acknowledgement, understanding of the underlying mechanisms and dynamics of social support is lacking. Efforts being made in the fields of mobile and ubiquitous computing (Ubicomp) to effectively harness social influence within health-related behavioural change applications have so far had variable success.

In a short term trial of a system that shared daily step counts and goal progress between friends, Consolvo et al [3] found that the trial group who shared their data were significantly more successful at achieving their goals than the control group who did not share their data with anyone. In contrast, a similar system that shared physical activity and nutritional intake was found to be no more effective than the single-user equivalent [4]. A longer-term trial of a step count-based game also found that the group who shared data and played as a team were no more successful than those who played alone [5]. There are many differences between the systems (manner of data capture and presentation) and their respective trials (duration and conditions), but an important difference to highlight is that it was only during the trial of the first system, in which social networks were found to be significant, were the people who shared data aware of and familiar with each other before the trial. This seems to suggest that existing peer groups benefit most from the inclusion of social support in such activity promotion applications.

Through our pilot-study of Shakra, a mobile phone-based application that detects the amount of time a person spends walking and shares that information between friends, we found that participants responded positively to the application and enjoyed looking at each others' daily activity levels [6]. Different groups of friends used it in different ways: one group used it as a game; constantly competing to beat each other's accumulated activity totals. The second group displayed less competitive tendencies, instead setting each other's activity levels within the context of the person undertaking the activity. The younger member of the group viewed one of her friends as a positive role-model, and so would set her own daily goals in relation to the activity total of her role-model; she would not compete with her friend but aimed 
instead to remain within a set amount of her friend's total minutes. The final group was not particularly interested in how much activity each other had accumulated but enjoyed the sense of awareness that the tool provided of each other when they were apart. Our study highlights the diverse nature of peer groups, and along with the findings discussed earlier, emphasises the need for a greater understanding of how, when, why and with whom people would like to share health behaviour-related information about themselves.

\section{Collabohab}

The aim of this work is to explore, in depth, the issues and factors of social support that are important to the cardiac rehab participants and explore the potential for use of mobile and communications technology in this domain. The study will focus on patterns of interaction exist between rehab participants, their rehabilitation physiotherapists and nurses, and specific members of their friends and family. A technology probe, called Collabohab, will be deployed into the lives of such peer groups for the duration of the rehabilitation program. Unlike Hutchinson et al's [10] technology probes, the physicality of Collabohab is not particularly provocative. However, in the same way that Gaver et al [11] advocate the use of low-tech everyday media, it is suggested that presenting novel and exploratory uses of commodity technology will also serve as a catalyst for interesting and informative responses.

Collabohab is a mobile-phone based multimedia rehabilitation journal that allows participants to share their experiences with their peer group. The mobile phone will allow the rehabilitation participants to monitor their behavioural and physiological risk factors (as currently written on a record card). Using the same activity inference techniques as Shakra [6], the amount of time that the carrier spends walking is monitored by the phone. The remaining behavioural (smoking, diet and alcohol intake) and physiological data (cholesterol, blood pressure, pulse and weight) can be manually entered by the rehab participant or nurse using the phone or a web-interface. Photographic, audio and video 'memoirs' can be captured and uploaded from the phone to the journal. A basic temporal representation of the participants' data will be accessible on the phone, and a web page will provide full visualisation of their journal entries and allow them to annotate their data. Only rehab participants will be given a phone. Using a web browser, rehab professionals and peers will be able to view the journal of the participant they know, for whom they can also leave messages. Rehabilitation participants will also be able to view and contribute to each other's journals.

Analysis of Collabohab's logs will help reconstruct patterns of use and interaction over the 10 week rehabilitation period. This quantitative data will be augmented with information gathered during in-depth interviews with all of the study participants performed at the beginning, mid-point and on completion of the rehabilitation program. Having been asked to capture 'things' relating to their rehab experience (e.g. people, places, events, etc); the multimedia memoirs will serve as a resource for discussion during the interviews. It is hoped that by taking this mixed quantative/qualitative approach we will learn not only about what interactions occurred through and around the probe, but about why those interactions happened. Is 
sharing data in this manner just another case of 'Big Brother' for the rehab participant or is it a valuable source of one dimension of social support: feeling cared for? The study will also generate important feedback on the usability and suitability of Collabohab as a supportive technology. Learning about Collabohab's failures and successes will guide the design of the next generation of supportive collaborative rehabilitation technology.

\section{Contribution to $\mathrm{HCI}$}

Two goals drive this research: we want to (1) generate insight into the social dynamics that surround behavioural change within a rehabilitation program and (2) investigate ways in which technology can be used to support and exploit these dynamics. While Shakra [6] marks preliminary and exploratory steps in this area, the study described in this paper aims to satisfy the first goal while continuing advances towards the second.

As interest in the HCI community surrounding the application of technology to health-related behavioural change increases, the need to ground such applications with knowledge of their intended domain grows. This work will contribute to the body of knowledge surrounding social influence within behavioural change applications and provide guidelines for the development of such systems within cardiac rehabilitation programs.

\section{References}

1. WHO, World Health Organisation (2006), http://www.who.org

2. McLean, N., et al.: Family involvement in weight control, weight maintenance and weightloss interventions: a systematic review of randomized control trials. International Journal of Obesity 27, 987-1005 (2003)

3. Consolvo, S., et al.: Design Requirements for Technologies that Encourage Physical Activity. In: CHI. 2006, Montreal

4. Gasser, R., et al.: Persuasiveness of a Mobile Lifestyle Coaching Application using Social Facilitation. In: IJsselsteijn, W., de Kort, Y., Midden, C., Eggen, B., van den Hoven, E. (eds.) PERSUASIVE 2006. LNCS, vol. 3962, Springer, Heidelberg (2006)

5. Lin, J.J., et al.: Fish'n'Steps: Encouraging Physical Activity with an Interactive Computer Game. In: Dourish, P., Friday, A. (eds.) UbiComp 2006. LNCS, vol. 4206, Springer, Heidelberg (2006)

6. Maitland, J., et al.: Increasing the Awareness of Daily Activity Levels with Pervasive Computing. In: Pervasive Computing Technologies for Healthcare, IEEE, Austria (2006)

7. Haughton McNeill, L., Kreuter, M., W., Subramanian, S., V.: Social Environment and Physical activity: A review of concepts and evidence. Social Science \& Medicine 63(4), 1011-1022 (2006)

8. Arthur, H.M.: Depression, isolation, social support, and cardiovascular disease in older adults. Journal of Cardiovascular Nursing 21(55), 52-57 (2006)

9. Berkman, L., Glass, T.: Social integration, social support, and health. In: Berkman, L., Kawachi, I. (eds.) Social Epidemiology, Oxford University Press, New York (2000)

10. Hutchinson, H., et al.: Technology Probes: Inspiring Design for and with Families. In: CHI. 2003 (2003)

11. Gaver, B., Dunne, T., Pacenti, E.: Design: Cultural probes. interactions 6(1), 21-29 (1999) 